\title{
ORIGINAL
}

\section{ESTIMACIÓN DE LA COBERTURA DE LAS VACUNACIONES SISTEMÁTICAS EN LA POBLACIÓN INFANTIL DE LAS ISLAS BALEARES (*)}

\begin{tabular}{l} 
Antònia Galmés Truyols (1), Joana Ripoll Amengual(1), Antonio Nicolau Riutort (1), Mercedes \\
Seguí Chinchilla (2), Alicia Magistris Sancho (3), Catalina Bosch Isabel (1), Margarita Portell \\
Arbona (1), Isabel Martí Alomar (1), Francisca González Porcel (1) \\
(1) Servei de Promoció de la Salut i Epidemiologia. Conselleria de Sanitat i Consum. Govern de les Illes Balears. \\
(2) Centre Insular de Menorca. Conselleria de Sanitat i Consum. Govern de les Illes Balears. \\
(3) Centre Insular d'Eivissa i Formentera. Conselleria de Sanitat i Consum. Govern de les Illes Balears. \\
(*) Trabajo realizado con una beca de la Conselleria d'Educació i Cultura del Govern de les Illes Balears. \\
\hline
\end{tabular}

RESUMEN

Fundamentos: Para un buen desarrollo de los programas de vacunación es primordial conocer la cobertura de vacunación. En las Islas Baleares la cobertura notificada era muy inferior a la del resto de España. El objetivo de este trabajo es la estimación de la cobertura de las vacunas incluidas en el calendario recomendado hasta los 18 meses de edad (4 dosis de polio oral, tétanos y difteria; 3 de tosferina; 1 de sarampión, rubeola y parotiditis).

Métodos: Estudio descriptivo, transversal, de base poblacional. Se calcularon los estimadores puntuales de distribución de frecuencias y los intervalos de confianza al 95\% (IC95\%) correspondientes, sobre una muestra de la población residente en Baleares de dos años de edad (nacida en 1995), seleccionada por muestreo aleatorio por conglomerados en una sola etapa a partir de las secciones censales. Se consultan los registros de vacunas de Sanidad y de los centros de salud del Insalud, se pide telefónicamente a las familias los datos del documento de vacunación y a los pediatras privados los de la historia clínica.

Resultados: La muestra incluía 606 niños. Pudo obtenerse la información completa de 532. Se consiguió la información completa de 377 casos $(62 \%)$ a partir de los registros del sector público. La cobertura más baja fue la de la vacuna antidiftérica, 518 niños complentamente vacunados, el 96,8\% (IC95\% = $94,8-98,1)$ y la más alta la de la vacuna antipertussis, 537 niños, el 98,9\% (IC95\% = 97,5 - 99,5).

Conclusiones: En las Islas Baleares existe una buena cobertura de la vacunación, similar a lo que se viene estimando en España en su totalidad. Se cumple el objetivo incluido en el Plan de Erradicación de la Polio. La información recogida desde el sector público es escasa.

Palabras clave: Vacunas. Poliomielitis. Difteria. Tétanos. Tos ferina. Sarampión. Rubeola. Parotiditis.

Correspondencia:

Antònia Galmés Truyols

Secció d'Epidemiologia. Conselleria de Sanitat i Consum.

Cecili Metel 18. 07003-Palma de Mallorca.

Correo electrónico agalmes@dgsanita.caib.es.
ABSTRACT

\section{Estimating the Coverage of Systematic Vaccinations among the Balearic Island Infant Population}

Background: In order for vaccination programs to be carried out properly, it is essential for the vaccination coverage to be known. On the Balearic Islands, the reported coverage was much less than for the rest of Spain. The objective of this study is that of estimating the coverage of the vaccinations included on the recommended schedule up to 18 months of age (4 doses of oral polio, tetanus and diphtheria; 3 doses of whooping cough; 1 dose of measles, rubella and mumps).

Methods: A descriptive, cross-sectional study of the population base. The ideal-time frequency distribution estimators and the corresponding confidence intervals were calculated at $95 \%$ (CI95\%) for a sample of the resident two-year-old (born in 1995) Balearic Island population selected by means of a single-stage conglomerate random sampling based on census sections. The National Public Health System and National Health Institute vaccination records were reviewed, the data from the vaccination document having been requested from the families and the clinical record from the private pediatricians by telephone.

Results: The sample included 606 children, it having been possible to obtain full information on 532 children. Full information was obtained on 377 cases $(62 \%)$ from the public health sector records. The least degree of coverage was found for the diphtheria vaccine, 518 children having been fully vaccinated, $96.8 \%(\mathrm{CI} 95 \%=94.8-98.1)$, the highest degree of coverage having been found for the whooping cough vaccine, 537 children, $98.9 \%($ CI95\% $=97.5-99.5)$.

Conclusions: Good vaccination coverage was found to exist on the Balearic Islands, being similar to what has been being estimated for Spain as a whole. The objective included within the Polio Eradication Plan is accomplished, very little information being gathered from the public sector.

Keywords: Vaccines. Poliomyelitis. Diphtheria. Tetanus. Whooping cough. Measles. Rubella. Mumps. 


\section{INTRODUCCIÓN}

Los programas de vacunación son un pilar de la política de salud pública de cualquier región o estado. Gracias a la inmunización vacunal se ha conseguido reducir de forma espectacular la morbi-mortalidad e incluso en el caso de la viruela eliminó la enfermedad y su agente causal ${ }^{1}$. Tras este éxito histórico, la Asamblea Mundial de la Salud fijó objetivos ambiciosos en ciertos problemas de salud. En 1988 planteó la erradicación del tétanos neonatal para el año 1995 y la de la poliomielitis para el $2000^{2}$ y en $1989^{3}$ añadió a sus objetivos la reducción del sarampión en un $95 \%$ de los casos.

La cobertura vacunal infantil en la Unión Europea en la primera mitad de los noventa era aceptable para polio, difteria-tétanos-tos ferina (DTP) y sarampión-rubeola-parotiditis (SRP), con coberturas en general superiores al $80 \%$, pero con notables diferencias entre países: Italia notificaba una cobertura del $50 \%$ en sarampión, Irlanda el $63 \%$ en polio, Alemania el $85 \%$ en DT y el $45 \%$ en tos ferina. Por otro lado Finlandia alcanzaba una cobertura prácticamente universal en polio y DTP, y del $98 \%$ para SRP ${ }^{4}$.

Una condición imprescindible en el Plan de Erradicación de la Poliomielitis era demostrar una buena cobertura de vacunación, algo que en las Islas Baleares no se había logrado. En 1994 en España se habían alcanzado coberturas del $88,1 \%$ para polio, $87,6 \%$ para DTP (definidas ambas como tres dosis a los 12 meses) y del $89,5 \%$ para SRP, en este caso una dosis entre 12 y 24 meses. Mientras, en las Islas Baleares se estaba lejos de alcanzar estas coberturas y en este mismo año la menor cobertura registrada era $50 \%$ para DTP y la mayor del $52 \%$ para SRP 5 . En 1997 las coberturas registradas en España eran ya del $94,4 \%$ para polio, $94,1 \%$ para DTP y del $93,1 \%$ para SRP, pero en las Islas Baleares fueron todavía, respectivamente, del $73,5 \%, 71,7 \%$ y $65,9 \%{ }^{6}$. Esta importante diferencia entre Baleares y el resto de España no concuerda con los indicadores indirectos de cobertura que se han venido manejando ${ }^{7}$ ni con la morbilidad por estas enfermedades en nuestro ámbito. Todo ello hacía necesaria la estimación de la cobertura real de vacunación, no sólo de polio sino de todas las vacunas incluidas en el calendario infantil. En una etapa anterior se compararon las dosis de vacuna de polio oral suministradas por el sector público (Conselleria de Sanitat al Insalud) y privado (laboratorios farmacéuticos a oficinas de farmacia) en el año 1996 con las dosis necesarias para cubrir a toda la población de 3, 5 y 7 meses, 6 y 14 años en el mismo año, y el resultado fue que el suministro excedía en 4.392 dosis. Por otra parte, los datos de morbilidad revelan que en las Islas Baleares la incidencia de las enfermedades controladas por las vacunas objeto del estudio es similar a la del resto de España ${ }^{8}$. Nuestra hipótesis era que se trataba de un problema de subregistro de vacunación y por ello hemos realizado un estudio poblacional, con el objeto de conocer las coberturas de las vacunas incluidas en el calendario recomendado en nuestra Comunidad Autónoma hasta los 18 meses de edad.

\section{SUJETOS Y MÉTODOS}

Se realizó un estudio descriptivo transversal, de base poblacional, a partir de la información de diversos registros de vacunación y de los padres de los sujetos incluidos. La población de estudio era la residente en las Islas Baleares que cumplió dos años de edad entre el 1 de enero y el 31 de diciembre de 1997, ambos incluidos. El estudio se realizó sobre la cohorte nacida durante el año 1995 empadronada en las Islas Baleares a 1 de enero de 1998, que según fuentes del Institut Balear d'Estadística (IBAE) era de 7.470 niños.

Para el cálculo de las estimaciones se seleccionó una muestra de la población objeto del estudio mediante muestreo aleatorio por conglomerados en una sola etapa ${ }^{9}$, en el cual la unidad de muestreo era la sección censal, 
con la inclusión en el estudio de todos los niños de la cohorte de las secciones seleccionadas. Se realizó la selección a partir del listado de las secciones censales (un total de 538) proporcionado por el Institut Balear d'Estadística (IBAE). La media de niños de la cohorte de nacidos en el año 1995 por sección censal fue calculada en 14 (7.470 niños / 538 secciones).

El cálculo del tamaño de la muestra se realizó teniendo en cuenta una proporción esperada de niños correctamente vacunados de 0,95 , para una población finita del tamaño de la cohorte (7.470 niños), para una precisión en las estimaciones de $\pm 1,7 \%$ y para un intervalo de confianza del $95 \%$. El resultado del cálculo fueron 528 niños, lo que representa incluir los niños de 37,7 secciones. Finalmente se decidió seleccionar aleatoriamente 40 secciones.

El listado de los niños incluidos y los datos necesarios para su localización se obtuvieron de los padrones municipales de población. Los datos que faltaban en los padrones se obtuvieron de la base de datos de la Tarjeta Sanitaria Individual del Insalud de Baleares. Esta misma fuente informó del pediatra del Insalud al cual estaban adscritos los niños.

Las definiciones utilizadas para conocer el estado de vacunación de cada niño se ela-

Tabla 1

Calendario de vacunaciones vigente en 1997 en la Comunidad Autónoma de las Islas Baleares hasta los 18 meses de edad

\begin{tabular}{|ll|}
\hline Vacuna & \multicolumn{1}{c|}{$\begin{array}{c}\text { Edad de } \\
\text { administración }\end{array}$} \\
\hline${\text { Polio } 1 .^{\mathrm{a}}}_{\text {Polio 2. }}^{\mathrm{a}}$ & 3 meses \\
Polio 3. & 5 meses \\
Polio 4. & 7 meses \\
Difteria-Tétanos-Tos ferina 1. & 18 meses \\
Difteria-Tétanos-Tos ferina 2. & 3 meses \\
Difteria-Tétanos-Tos ferina 3. & 5 meses \\
Difteria-Tétanos & 7 meses \\
Sarampión-Rubeola-Parotiditis & 18 meses \\
\hline
\end{tabular}

boraron a partir del calendario de vacunaciones oficial de la Comunidad Autónoma para 1997 (tabla 1) y son las siguientes:

- Vacunación completa: niño que ha recibido toda la serie de la vacuna (cuatro dosis para polio y DT, tres para tos ferina y una para SRP) a los 2 años de edad.

- Vacunación incompleta: niño que ha recibido alguna dosis, sin que se haya llegado a completar la serie.

- No vacunación: niño que no ha recibido ninguna dosis de la vacuna.

Se utilizaron las mismas definiciones para cada una de las vacunas excepto para SRP, para las que no procede la categoría «vacunación incompleta». Se realizó una estimación independiente para cada vacuna.

Se definió la «falta de respuesta» como niño sin registro de vacunas, o con registro incompleto, y a cuya familia resultó imposible entrevistar para conocer el estado real de la vacunación, es decir, los niños no localizados.

La cobertura de vacunación se calculó sobre las fichas con la información completa, es decir, excluyendo del denominador las faltas de respuesta, y se calculó independientemente para cada una de las vacunas.

Aparte de los datos de identificación de los niños, se recogieron el número de dosis recibidas de cada una de las vacunas incluidas en el calendario y fecha de administración. La estrategia de recogida de la información sobre vacunaciones fue la siguiente: en primer lugar se consultó el registro de vacunas de la Conselleria de Sanitat. Para completar la información se accedió a los registros asistenciales del Insalud a través de una solicitud a las enfermeras de pediatría de los centros de salud. Cuando con estas dos fuentes la información seguía siendo incompleta se contactó por teléfono con las familias de los niños y se les pidieron los datos consignados en el documento de vacuna- 
ción. En algunos casos en que la familia no tenía el documento se les pidió el nombre y teléfono del pediatra que había realizado la vacunación y se contactó para obtener los datos. En la tabla 2 se aporta la información recogida por fuentes.

Tabla 2

Distribución de la información recogida por fuente

\begin{tabular}{|c|c|c|c|}
\hline Fuente de información & Número de niños & Porcentaje & Porcentaje acumulado \\
\hline Registro de vacunas Conselleria de Sanitat & 248 & 40,9 & 40,9 \\
\hline INSALUD & 46 & 7,6 & 48,5 \\
\hline R. Sanitat y INSALUD ${ }^{1}$ & 83 & 13,7 & 62,2 \\
\hline R. Sanitat y Doc. vacunación ${ }^{1}$ & 37 & 6,1 & 68,3 \\
\hline R. Sanitat y Pediatra sector privado ${ }^{1}$ & 3 & 0,5 & 68,8 \\
\hline INSALUD y Doc. vacunación ${ }^{1}$ & 8 & 1,3 & 70,1 \\
\hline Documento de vacunación & 101 & 16,7 & 86,8 \\
\hline Pediatra sector privado & 9 & 1,5 & 88,3 \\
\hline Información sin completar ${ }^{2}$ & 71 & 11,7 & 100,0 \\
\hline Total incluidos en la muestra & 606 & & \\
\hline
\end{tabular}

${ }^{1}$ Para completar la información se recogieron datos de ambas fuentes. El orden de consulta fue el que aparece en la tabla.

${ }^{2}$ Casos sin información completa para alguna vacuna. En algunas ocasiones está correctamente registrada alguna vacuna pero no otra,, y no se ha podido contactar con la familia para obtener la información.

El análisis estadístico se realizó con la ayuda de los programas informáticos Epiinfo 6.04 (CDC Atlanta, USA) para cálculo del tamaño de la muestra, selección aleatoria de los conglomerados y SPSS v. 8 para los cálculos. Se calcularon los estimadores puntuales de frecuencias relativas y los intervalos de confianza al 95\% (IC95\%) correspondientes.

\section{RESULTADOS}

Las 40 secciones censales incluidas correspondían a 13 municipios de Mallorca (con 15 secciones de Palma, la capital), tres de Menorca y uno de Ibiza. El número final de niños fue un poco más elevado que el calculado puesto que los padrones se actualizan permanentemente y siempre existen diferencias entre éstos y el censo. Así pues, la muestra que se estudió incluía 606 niños, de los cuales 314 eran varones. Su distribución por islas fue la siguiente: el $82 \%$ de Mallorca, el $14 \%$ de Menorca y el $4 \%$ de Ibiza y Formentera; los niños de Palma, que con- centra el $44 \%$ de niños de esta edad de las Baleares, representan el $40 \%$ del total de la muestra.

En el caso de Palma se obtuvo un listado conjunto de todos los niños de las secciones solicitadas en el que no constaba la sección de cada uno de ellos. En cambio sí constaba el distrito postal, y se utilizó esta variable para estudiar la distribución de las faltas de respuesta en la capital. En el resto de la Comunidad se utilizó la información sobre secciones.

Se obtuvo la información completa de 532 niños de los 606 incluidos, es decir el $88,3 \%$. En la tabla 2 se recoge la distribución por fuentes de información, y en ella destaca que solamente se pudo completar el $41 \%$ de las fichas utilizando exclusivamente los datos del Registro de la Conselleria de Sanidad, y se alcanzó el $62 \%$ añadiendo a éstos los aportados por el Insalud.

La vacunación antipoliomielítica era completa en 520 casos, el 97,2\% (IC95\% = 
$=95,1-98,2 \%)$ y los 17 casos restantes habían recibido al menos una dosis de vacuna. Para el resto de vacunas la cobertura menor fue la de la antidiftérica, con el $96,8 \%$
(IC95\% $=94,8-98,1 \%)$ y la mayor la de la antipertussis, con el 98,9\% (IC95\% = 97,5$99,5 \%)$. Los resultados se presentan en la tabla 3.

Tabla 3

Distribución de los niños con información completa por número de dosis recibidas y proporción de vacunados correctamente

\begin{tabular}{|c|c|c|c|c|c|c|c|c|}
\hline & \multicolumn{5}{|c|}{ Número de dosis } & \multirow{2}{*}{ Total niños ${ }^{I}$} & \multirow{2}{*}{$\begin{array}{c}\text { Vacunación } \\
\text { correcta }\end{array}$} & \multirow{2}{*}{ IC 95\% } \\
\hline & 0 & 1 & 2 & 3 & 4 & & & \\
\hline Polio & 0 & 1 & 1 & 14 & 520 & 536 & $97,0 \%$ & $95,1-98,2$ \\
\hline Difteria & 1 & 2 & 1 & 13 & 518 & 535 & $96,8 \%$ & $94,8-98,1$ \\
\hline Tétanos & 0 & 2 & 1 & 13 & 519 & 535 & $97,0 \%$ & $95,1-98,2$ \\
\hline Tos ferina & 2 & 2 & 2 & 537 & - & 543 & $98,9 \%$ & $97,5-99,5$ \\
\hline Sarampión & 12 & 525 & - & - & - & 537 & $97,8 \%$ & $96,0-98,8$ \\
\hline Rubeola & 11 & 526 & - & - & - & 537 & $97,9 \%$ & $96,2-98,9$ \\
\hline Parotiditis & 12 & 525 & - & - & - & 537 & $97,7 \%$ & $96,0-98,8$ \\
\hline
\end{tabular}

${ }^{1}$ Este denominador es el total de niños para los que la información pudo completarse. No coinciden por completo para cada una de las vacunas puesto que en algunos casos estaban registradas todas las dosis de una vacuna y otras no, pero no se pudo completar la información. Conocemos, pues, la situación de alguna de las vacunas pero no de otras.

Las faltas de respuesta (tabla 4) fueron 68 , el $12 \%$ del total. La mayor pérdida de información tuvo lugar en Palma (45 niños no localizados de un total de 2.261); la falta de respuesta en la capital se presentó en las zonas correspondientes a 10 de los 12 distritos postales incluidos en las 15 secciones. En el resto de la Comunidad las faltas de respuesta (29 niños) se distribuyeron en 10 de las 25 secciones; destaca la elevada proporción (99\%) de niños de Menorca cuya información se ha podido completar. El $43 \%$ de los niños del grupo de faltas de respuesta (no localizados) tenía alguna dosis documentada para polio, el $37 \%$ para DT y el $21 \%$ para tos ferina.

\section{DISCUSIÓN}

Los resultados del estudio confirman la buena cobertura de la vacunación en Baleares, similar a lo que se viene estimando en España en su totalidad ${ }^{10}$ y en alguna Comunidad Autónoma ${ }^{11}$. Las coberturas notifica- das para Baleares son muy bajas y en cambio las estimadas en el presente estudio son muy aceptables. Son varias las razones que podrían justificar esta aparente contradicción, la principal de las cuales podría ser el gran peso que la asistencia médica privada tiene en nuestra Comunidad, ya que este sector en el año en que se realizó el estudio no estaba obligado a notificar a Salud Pública las vacunaciones que efectuaba. Por otra parte se detectaron deficiencias de notificación de las vacunas administradas en los centros de salud del Insalud y, por último, el programa informático de gestión del Programa de Vacunaciones reveló una serie de problemas que dificultan un correcto registro y una buena elaboración de los datos. Hay en las Baleares una numerosa población flotante, distinta a la turística, que pasa unos meses al año en la Comunidad por motivos de trabajo de algún miembro de la familia, sin que se sepa si este hecho motiva una estimación de la cobertura desviada de la real y, si así fuera, se desconoce si supondría una infra o una supraestimación. 
Tabla 4

Distribución geográfica de los niños: número total, registros con información completa y faltas de respuesta

\begin{tabular}{|c|c|c|c|}
\hline & $\begin{array}{l}\text { Número de niños } \\
\text { y porcentaje }\end{array}$ & Categoría $^{I}$ & Número de niños \\
\hline $\begin{array}{l}\text { Palma } \\
\text { (capital) }\end{array}$ & $261(43 \%)$ & $\begin{array}{l}\text { información completa } \\
\text { faltas de respuesta }\end{array}$ & $\begin{array}{l}216(83 \%) \\
45\end{array}$ \\
\hline $\begin{array}{l}\text { Resto de } \\
\text { Mallorca }\end{array}$ & $258(39 \%)$ & $\begin{array}{l}\text { información completa } \\
\text { faltas de respuesta }\end{array}$ & $\begin{array}{l}235(86 \%) \\
23\end{array}$ \\
\hline Menorca & $84(14 \%)$ & $\begin{array}{l}\text { información completa } \\
\text { faltas de respuesta }\end{array}$ & $\begin{array}{l}83(99 \%) \\
1\end{array}$ \\
\hline $\begin{array}{l}\text { Ibiza y } \\
\text { Formentera }\end{array}$ & $25(4 \%)$ & $\begin{array}{l}\text { información completa } \\
\text { faltas de respuesta }\end{array}$ & $\begin{array}{l}21(81 \%) \\
5\end{array}$ \\
\hline Total & $606(100 \%)$ & $\begin{array}{l}\text { información completa } \\
\text { faltas de respuesta }\end{array}$ & $\begin{array}{l}532(88 \%) \\
74\end{array}$ \\
\hline
\end{tabular}

(1) Las faltas de respuesta se definieron como niño sin registro de vacunas, o con registro incompleto, y a cuya familia resultó imposible entrevistar para conocer el estado real de la vacunación, es decir, los niños no localizados.

Los niños con información completa son aquellos con toda la información registrada, o aquellos cuya información pudo completarse al contactar con la familia.

Las coberturas observadas en el presente estudio, al igual que las del resto de Espa$\tilde{\text { na }}{ }^{4}$, están en el tramo de las más altas de los países de la Unión Europea ${ }^{3}$. Puede afirmarse que hay un buen nivel de inmunidad colectiva de los niños de esta cohorte, con una probabilidad mucho más baja de transmisión de la enfermedad a los escasos individuos susceptibles por la interrupción de la cadena epidemiológica. Evidentemente esta protección, para tasas de cobertura iguales, es tanto mayor cuanto mayor sea la efectividad de la vacuna, y así se evidencia en los brotes de parotiditis que han ocurrido en poblaciones con tasas de vacunados muy elevadas, pero con una vacuna cuya eficacia resultó ser muy baja ${ }^{12}$. En el caso del tétanos, cuya protección depende exclusivamente de la vacunación individual, no se puede hablar de inmunidad colectiva, pero en cualquier caso la cobertura es muy elevada. En el desarrollo de programas de control de las enfermedades seroprevenibles deben ser tenidos en cuenta la eficacia de la vacunación y la existencia o no de inmunidad colectiva.

En el caso de la polio se cumple el objetivo incluido en el Plan de Erradicación, que es el de alcanzar una cobertura suficiente, definida por la OMS como un mínimo provincial del $95 \%$. 13 .

La cobertura estimada en sarampión permite también ser optimista en cuanto al cumplimiento de los requisitos exigidos para la inminente puesta en marcha del Plan de Erradicación, es decir, conseguir con la vacunación que la población susceptible no supere el $5 \%{ }^{14}$.

El objetivo de la vacunación antirrubeólica es la eliminación de la infección prenatal, por lo que la elevada cobertura en la cohorte estudiada no tiene una incidencia directa sobre el efecto deseado - la protección de las mujeres gestantes - aunque sí puede influir indirectamente el estrecho contacto de estos niños con mujeres en edad fértil, que verán disminuida la probabilidad de infección si sus fuentes potenciales de contagio están inmunizadas.

La experiencia de algunos países en los que por causas diversas ha disminuido la cobertura de la vacunación infantil demuestra que es imprescindible mantener nuestra actual cobertura. Los Nuevos Estados Independiente de la antigua URSS, cuya penuria 
económica ha impedido mantener los programas de vacunación existentes, han visto reaparecer la poliomielitis, y en estos mismos estados y alguno limítrofe con ellos (Polonia) ha reaparecido la difteria.

Algunas comunidades que por razones ideológicas o religiosas rehusan la vacunación (Canadá 1991, Holanda 1992) ${ }^{15}$ y algunos colectivos que no alcanzan las coberturas deseadas (gitanos en Bulgaria en 2001) ${ }^{16}$ han padecido brotes de poliomielitis. La creciente negativa a vacunar por creencias personales, que todavía no parece haber alcanzado proporciones preocupantes en nuestro medio, el incremento de los viajes a países donde la circulación de los agentes causales de estas enfermedades todavía es muy alta y la recepción de inmigrantes con coberturas de vacunación insuficientes procedentes de estos mismos lugares deberían constituir una alerta para nuestros programas de vacunación, de forma que se asegure su capacidad para identificar y vacunar a los niños de estos colectivos.

En nuestro estudio se recogieron las razones de la vacunación incompleta o no vacunación, y sólo en un caso se debía a una negativa de los padres, otro a contraindicación médica, uno a una adopción internacional que había empezado la vacunación con retraso y el resto a padres que habían olvidado visitar al pediatra para la actualización del calendario de vacunación, generalmente para las vacunas que se administran en las edades más avanzadas (SRP, cuarta de polio y de DT). La desaparición del tétanos en edades infantiles y de la difteria y la poliomielitis, junto con la baja incidencia del resto de enfermedades en nuestro medio, la escasa percepción de gravedad que se tiene de las mismas y la confianza en los tratamientos médicos probablemente influyan en estos olvidos. Posiblemente esta sensación de seguridad también influya en el aumento de los grupos contrarios a la vacunación. Por otra parte, no hay que menospreciar la magnificación mediática de los efectos secundarios de las vacunas y de las falsas contraindicaciones, que podrían influir en disminuciones de la cobertura $^{17}$.

Las faltas de respuesta (12\%), que en este estudio están constituidas por los niños sin información suficiente por no constar en el registro ni haber sido posible localizarlos, no parecen excesivas para un diseño de estas características, de base poblacional a partir de los padrones municipales y en una población con una importante movilidad. A pesar de que es siempre de difícil interpretación el posible sesgo debido a las faltas de respuesta, el hecho de que no se concentren en unos lugares determinados hace pensar que no se trata de una población esencialmente diferente de la que ha aportado la información. Tampoco se ha visto una mayor frecuencia de falta de respuesta en barrios con alguna característica social o económica que permita pensar que sus habitantes tengan menor probabilidad de recibir las vacunas recomendadas. En Palma (con unos trescientos mil habitantes) la falta de respuesta es más alta que en el resto de municipios (el mayor de los cuales tiene unos treinta mil habitantes), cuya información es siempre más accesible que en las grandes ciudades.

Aunque el objetivo del estudio no fuera el análisis del Registro de Vacunaciones de Sanidad, y por tanto los hallazgos no puedan presentarse como estimadores de la situación, es evidente que presenta importantes deficiencias. Por ello debería incrementarse la notificación de vacunaciones desde el sector público e implementarse un sistema de registro eficaz para las realizadas por el sector privado. Probablemente esta situación indique un problema de cultura en salud pública entre los profesionales asistenciales. En las Islas Baleares se ha legislado específicamente sobre la materia ${ }^{18}$, aunque probablemente ello no sea suficiente para conseguir buenos registros. Es necesaria una mayor implicación de las autoridades sanitarias, de los técnicos de salud pública y de los docentes de las escuelas de Enfermería y facultades de Medicina, así como de 
los de la formación de postgrado, para que estos profesionales comprendan la dimensión colectiva de su trabajo. Por el contrario, los documentos de vacunación que guardan los padres constituyeron una excelente fuente de información.

Finalmente, es de destacar la escasa bibliografía relativa a cobertura poblacional de vacunación en los países desarrollados. Salvo el estudio de seroprevalencia (que, aunque recoja la información sobre vacunación, no es un estudio de cobertura, sino de inmunidad) realizado por el Centro Nacional de Epidemiologia ${ }^{8}$ en 1996, que incluye toda España excepto Cataluña, y el estudio de cobertura de 1998 en Galicia ${ }^{9}$, el resto de artículos se refieren a grupos muy concretos (grupos sociales con problemas específi$\cos ^{19}$, población infantil hospitalizada ${ }^{20}$, estudios en el contexto de la investigación de brotes epidémicos ${ }^{21}$, en escolares de un área $^{22}$ ). Lo mismo ocurre en el resto de países desarrollados. Esta falta de interés en la difusión de resultados de programas posiblemente también responda a la sensación de seguridad a que nos referíamos anteriormente, en este caso dando por supuesto que tenemos una cobertura excelente. Consideramos que estudios como el presentado son muy útiles para estimar coberturas cuando no existe un registro fiable, o cuando su puesta en marcha y funcionamiento son demasiado costosos. Cuando éste existe, los estudios de cobertura sirven también para evaluar el programa de vacunaciones y el funcionamiento del registro, identificar grupos de baja cobertura, identificar los motivos de la falta de vacunación, y en general obtener información útil para la planificación.

\section{AGRADECIMIENTOS}

Los autores agradecen la colaboración del Institut Balear d'Estadística (Joan Pons), Gerencia de Atención Primaria del Insalud de Mallorca (Mateu Mesquida, Joan Llobera y Carlos Campillo), Centros de Salud del
Insalud y las secciones de Programas Sectoriales y Materno-Infantil de la Conselleria de Sanitat.

\section{BIBLIOGRAFÍA}

1. Salmerón García F. Las enfermedades sometidas a programas de vacunación. Aspectos epidemiológicos y preventivos. En: Martínez Navarro F: Salud Pública, Madrid: Mc.Graw-Hill Interamerinaca; 1998. p. 477.

2. Resolución WHA 41.28. Global eradication of poliomyelitis by the year 2000. Organización Mundial de la Salud. (http://policy.who.int/ cgi-bin/om_isapi.dll?infobase=WHA\&softpage $=$ Browse_Frame_Pg42).

3. Resolución WHA 42.32. Expanded Programme on Immunization. Organización Mundial de la Salud. (http://policy.who.int/cgi-bin/om_isapi.dll?infobase=WHA\&softpage=Browse_Frame_Pg42).

4. Guérin N, Roure C, Couverture vaccinale dans l’Union Européenne. Eurosurv 1997; 2: 2-4.

5. Regidor E, Gutierrez-Fisac JL. Indicadores de Salud. Cuarta evaluación en España del Programa Regional Europeo Salud para todos. Madrid: Ministerio de Sanidad y Consumo; 1996.

6. Conselleria de Sanitat i Consum. Secció d'Epidemiologia. Fulls setmanals de Vigilància Epidemiològica, núm. 13/98..

7. Centro Nacional de Epidemiología. Bol Epidemiol Sem 1997; 5 (1): 7-10.

8. Silva Ayçaguer, LC. Muestreo para la Investigación en Ciencias de la Salud. Madrid: Ediciones Díaz de Santos, SA; 1993. p. 87-97.

9. Pachón del Amo I. Situación del sarampión en España. Estudio seroepidemiológico. Rev Esp Salud Pública 1999; 73: 609-616.

10. Rego E, Santiago I, Zubizarreta R, Malvar A, Hervada X, Fernández S. Cobertura vacunal en Galicia 1998. Vacunas. Investi prác 2000; 1: 10-14.

11. Pons C, Pelayo T, Pachón I, Galmés A, González L, Sánchez C, Martínez F. Deux épidemies d'oreillons chez des enfants vaccinés avec la souche Rubini en Espagne indiquent une faible efficacité vaccinale. Eurosurv 2000; 5: 80-84.

Rev Esp Salud Pública 2002, Vol. 76, N. ${ }^{\circ} 4$ 
12. Ministerio de Sanidad y Consumo Plan de actuaciones necesarias para la consecución del certificado de erradicación de la poliomielitis. Madrid: Ministerio de Sanidad y Consumo; 1997.

13. Instituto Carlos III Plan de Eliminación del Sarampión en España. Madrid: Instituto Carlos III; 2000.

14. Gestal Otero J, Rodríguez Mínguez L, Montes Martínez A, Takkouche B. Emergencia en Europa de la difteria y la poliomielitis. Rev Esp Salud Pública. 1996; 70: 5-14.

15. WHO. Imported wild poliovirus causing poliomyelitis, Bulgaria, 2001,. Wkly Epidemiol Rec 2001; 76(43): 332-5 (http://www.who.int/wer/pdf/2001 /wer7643.pdf).

16. Gellin BG, Maibach EW, Marcuse EK, ¿Los padres conocen las vacunaciones? Una encuesta telefónica a escala nacional. Pediatrics (ed. esp) 2000; 50: 355-6.
17. Ordre de la Conselleria de Sanitat i Consum, de dia 2 d'octubre de 2000, per la qual es regula l'accés gratuït a les vacunes incloses en el Calendari de vacunes infantil de les Illes Balears.

18. Ubierna Díez J. Estudio sobre el estado de vacunacion en poblacion gitana menor de 14 años adscrita a un centro de salud en Burgos. Centro de Salud 1997; 5: 236-240.

19. Hernandez Latorre M A, Villalbi Hereter J, Vall Combelles O, Grauges Pons D, Rovira Negre G, Martinez Roig A. Estado vacunal en pacientes pediátricos ingresados. Act Pediatr Esp 1997; 55 (3): 110-4

20. Amela C, Pachon I, Ibáñez C. Medidas del efecto de la vacunación en un brote de sarampión. Gac Sanitaria 1993; 7: 164-8.

21. Albertos MA, Rovira G, Villalbí JR. Cobertura vacunal en escolares. Rev Esp Salud Pública 1991; 65: 269-275. 